\title{
The Proof of Mass Decay
}

\author{
Dr. Andreas Gimsa \\ Stirling Technologie Institut gemeinnützige GmbH, D-14478 Potsdam, Germany
}

\begin{abstract}
It is high time that scientists recognise the general mass decay of all objects in the universe. Otherwise, the process of physical knowledge will remain severely limited. For the world is changeable and existing material structures, including the masses, must change with it. Change presupposes a flow of time, which in turn is influenced and caused by matter [1.].
\end{abstract}

Keywords: Mass decay, Expansion of Universe, Conservation of Momentum, Time, Entropy

\section{Introduction}

This publication is intended to prove mass decay and to explain why an accelerated expansion of space is assumed today, but this cannot occur. It is shown that under the conditions of mass decay, the conservation of momentum applies. In addition, it is shown that gravity only functions in the observable form with a constant number of effects, as is also the basis of mass decay. This is demonstrated using a two-mass system.

\section{Atomic Clocks}

As explained in the publication "The Metric of Space-time" [1.], all masses, distances, frequencies, periods and the gravitational value change with the age of space. The resonance frequency of each atomic clock becomes lower with time. The regulator of the atomic clock, which adjusts the frequency of the microwave resonator (e.g. 9,192,631,770 Hz) according to the resonance frequency, finds the change of the energetic atomic states at lower and lower frequencies. The second is then assigned to an ever lower frequency value. At the same time, however, the period of the individual oscillation has increased. This leads to the fact that the calibrated second remains constant with the space age. All other natural periods, on the other hand, become longer over time and all frequencies lower.

\section{Distance Measurement}

A changing distance from year to year, e.g. the increase in distance of the moon in relation to the earth, can be measured reliably because the light travel time is measured with a constant seconds scale of the atomic clock. The distance increases, the light now requires more seconds kept constant and the distance can be calculated. If the time scale of the atomic clock, like all other natural periods, were to increase with the expansion of space, the expansion-related increase in the distance between the Earth and the Moon could not be measured [2.]

\section{Measurement of the Redshift}

The redshift $z$ can be determined according to the known formula from the reference frequency in the near distance $f_{0}$ and the observed frequency $f_{o b s}$ in the far distance:

$$
z=\frac{f_{0}}{f_{o b s}}-1
$$


For the frequency measurement of an observer, according to the Doppler effect for the medium of light, the geometric mean of 1 . the falling frequency $f_{o b s 1}$, which a theoretically stationary observer measures at a

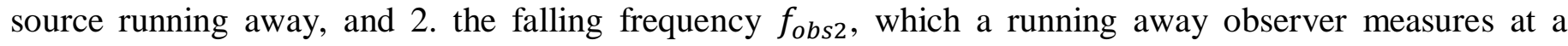
theoretically stationary source, applies:

$$
f_{\text {obs }}=\sqrt{f_{o b s 1} f_{o b s 2}}=\sqrt{\frac{1}{1+\frac{v}{c}} f_{0}\left(1-\frac{v}{c}\right) f_{0}}=f_{0} \sqrt{\frac{c-v}{c+v}}
$$

Furthermore, for the frequency measurement of an observer according to the Doppler effect for the medium light, the geometric mean of 1 . the increasing frequency $f_{o b s 3}$ measured by a theoretically stationary observer at an incoming source and 2. the increasing frequency $f_{o b s 4}$ measured by an incoming observer at a theoretically stationary source applies:

$$
f_{o b s}=\sqrt{f_{o b s 3} f_{o b s 4}}=\sqrt{\left(1+\frac{v}{c}\right) f_{0} \frac{f_{0}}{1-\frac{v}{c}}}=f_{0} \sqrt{\frac{c+v}{c-v}}
$$

These well-known equations (02) and (03) result without the special theory of relativity.

If the light source and observer move away from each other, equation (02) applies; if the light source and observer approach each other, equation (03) applies.

From equation (02) follows with (01) the well-known calculation formula for the velocity-conditioned redshift:

$$
z=\frac{f_{0}}{f_{o b s}}-1=\sqrt{\frac{c+v}{c-v}}-1
$$

It is assumed that our universe is spherical and that its radius results from its age multiplied by the speed of light. The expansion of the universe does not proceed at an accelerated rate, but in such a way that its radius $r_{u n i}$ increases proportionally with the age $t_{\text {uni }}$ [3.]. The distances between the galaxies increase proportionally with the age of the universe. Thus the radial velocities of the galaxies always remain constant with space as it expands. As the galaxies move away from each other, there must be a velocity-induced redshift according to equation (04). Galaxies that are far away have a larger redshift [4.]. In a spherical universe whose galaxies are equally distributed, it is probable that our galaxy is located in the significantly larger volume area of the outer half of the radius. The probability of this is $87.5 \%$ [3.]. If, for example, the velocity-related redshift of a galaxy is $z=8.55$, as has already been measured, this would mean according to (04) that this galaxy is moving away from us at $97.8 \%$ of the speed of light in relation to ours. Those galaxies that are located near an imaginary outer sphere of the universe are particularly fast. This is because the radius of the universe grows at the speed of light, while its centre has no speed. If our galaxy is on the outside of the universe, it has a high velocity with space that is greater than $50 \%$ of the speed of light. If a distant galaxy is also moving in the outer area with the opposite direction and more than $50 \%$ of the speed of light away from us, both speeds add up and the distant galaxy remains invisible to us because the resulting speed is greater than the speed of light.

If we were to move away from the centre of space at $50 \%$ the speed of light, and another galaxy were to move away from the centre in the opposite direction at $47.8 \%$ the speed of light, then the resulting speed measured at our location would be $97.8 \%$ the speed of light with redshift $z=8.55$. Of course, it would also be possible for us to move with space at $98 \%$ the speed of light and for the distant galaxy to move in the same direction at $0.2 \%$ the speed of light.

Much more likely, however, is the arbitrary movement of another galaxy, which leads away from ours at a certain angle and the resulting relative speed of both galaxies is $97.8 \%$ of the speed of light. It should be possible to determine the centre of the universe, since the greatest redshifts should occur in this direction. 
However, this presupposes that we are actually in the outer region of the universe and that the measured velocities of the galaxies are those that can be assigned to the expansion of space.

Figure 1 shows the universe with exemplary galaxies. It can be seen that the galaxy's location in the universe determines its velocity with space. Towards the outside, the velocities increase up to the speed of light directly on the radius line. If one measures the Hubble constant, the value $70.9 \mathrm{~km} /(\mathrm{s} \mathrm{MPc})$ must come out in this model for all locations. The largest visible distance is 4,228 MPc. The reciprocal Hubble value in SI units corresponds to the space age, since galaxies at this distance must move at the speed of light relative to us. And now it gets exciting: the ratio of the distance to the speed of any galaxy in relation to ours always corresponds to the ratio of the space radius to the speed of light at all locations. In the same way, one could measure the ratio of any galaxy in relation to another. If one measures the relative speed of a galaxy and its distance, the Hubble constant (the space age) must inevitably come out. That is, space is not expanding at all at an accelerated rate. Mr. Hubble and we have been wrong all along. In a spherical universe, the velocities of the outwardly increasing space shells simply increase from the resting centre up to the speed of light (see Figure 1).
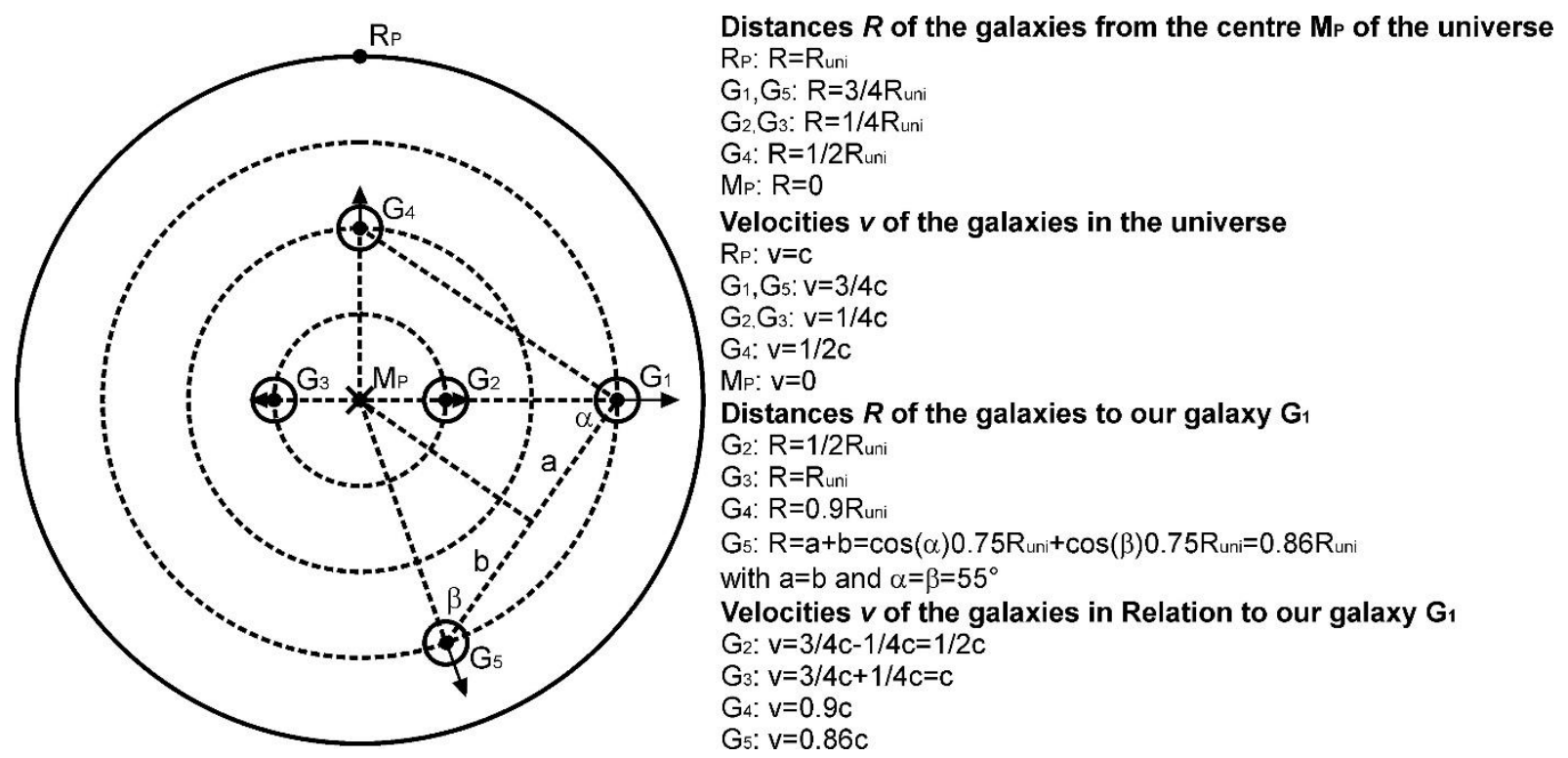

Figure 1: Our spherical universe with galaxies, their distances and velocities (Section through the centre, the galaxies $G_{1}$ to $G_{5}$ lie in the section plane)

The universe does not have to expand at an accelerated rate just because the velocity-induced redshift increases with distance. The radial velocities always remain constant and are simply greater in the outer region of the universe than in the inner.

As the universe ages, its masses decay. The equation of the constant entropy of the universe $S_{u n i}=$ $\frac{k}{\hbar} m_{u n i} t_{u n i} c^{2}$ is unrestrictedly valid and consistent with the observed redshift [5.].

The universe is a closed, thermally insulated system. Star radiation cannot reach the outside. It is absorbed and emitted by the outer spherical horizon. A radiation equilibrium is established between stellar radiation and horizon radiation. This is the observed $3 \mathrm{~K}$ background radiation. With this approach, the number of stars in the universe could be calculated in accordance with the star count [5].

One could still assume a gravitationally induced red shift, since the masses and gravitational field strengths were greater in the past. The coefficient $\frac{\gamma m}{r c^{2}}$ is contained in the calculation formula of the known gravitational redshift:

$$
z_{\text {grav }}=\frac{1}{\sqrt{1-\frac{2 \gamma m}{r c^{2}}}}-1
$$


The value $\gamma$ increases quadratically with space age, the mass falls and the radius increases with age. As a result, the coefficient remains exactly constant. This means that this temporal change in the three physical quantities has no influence on the measurement of the gravitationally induced redshift.

\section{Mass Decay and Conservation of Momentum}

Mass decay has no influence on the radial velocities of the galaxies as space expands. No force acts on the masses, and the radial velocities with space remain constant. With reference to the centre of a spherical universe, the radial velocity of a galaxy cannot be greater than the speed of light. This is because the radius of the universe grows exactly with the speed of light and the centre rests as a reference point for all galaxies [3.]. The relative speed of two galaxies moving in exactly opposite directions and theoretically located on the imaginary enveloping sphere of the universe would be exactly twice the speed of light. Two observers on each of these galaxies could therefore not perceive each other.

But how does mass decay affect the velocities of previously accelerated galaxies in space? The well-known conservation of momentum must apply here.

A force is generally defined as a change in momentum over time:

$$
F=\frac{d p}{d t}=\frac{d(m \vec{v})}{d t}
$$

Already in [7.] I have set up the following differential equation for mass in connection with mass decay and proved its validity:

$$
m=-\frac{1}{c^{2}} \frac{d m}{d t} \frac{d A}{d t} \quad \text { oder } \quad \frac{d m}{d t}+\frac{m c^{2}}{d A / d t}=\frac{d m}{d t}+\frac{E}{I}=0
$$

From this, the simple relation between mass and time was derived for the object:

$$
m_{0} t_{0}=m_{b} t_{b} \quad\left(N \hbar=m t c^{2}=\text { const. }\right)
$$

The basic relation can be understood as the principle of the constant number of effects. It means that while a certain time passes on the mass at rest, a smaller time passes on the moving (larger) mass. Or in the case of mass decay: If the mass becomes smaller, more time passes on it in relation to its mass at rest.

If there is a universal mass decay, then equation (06) must be differentiated according to the product rule. This is because we are dealing with two temporally variable quantities:

$$
\frac{d(m \vec{v})}{d t}=v \frac{d m}{d t}+m \frac{d \vec{v}}{d t}
$$

For the conservation of momentum to be valid, the force and consequently the change in momentum must be zero:

$$
0=v \frac{d m}{d t}+m \frac{d \vec{v}}{d t} \quad \text { or just like that } \quad 0=\vec{v} d m+m d \vec{v}
$$

The information-time equivalent was found and justified by me [8.]:

$$
I=t c^{2}
$$

From equation (07) follows with (11) the important and astonishing expression:

$$
\frac{d m}{m}=-\frac{d t}{t} \quad \text { or } \quad d m=-m \frac{d t}{t}
$$

Substituting equation (12) into (10) leads to the following equation:

$$
0=-\vec{v} m \frac{d t}{t}+m d \vec{v}=-\vec{v} \frac{d t}{t}+d \vec{v}
$$

Rearranging a little, we get the easily integrable formula: 


$$
\frac{1}{t} d t=\frac{1}{v} d \vec{v}
$$

The integration is to take place between a first $t_{0}$ and a subsequent age $t_{b}$ of the mass, i.e. between two age states:

$$
\int_{t_{0}}^{t_{b}} \frac{1}{t} d t=\int_{v_{0}}^{v_{b}} \frac{1}{\vec{v}} d \vec{v}
$$

From the integration, the following solution with the natural logarithm is obtained:

$$
\left.\ln t\right|_{t_{0}} ^{t_{b}}=\left.\ln \vec{v}\right|_{v_{0}} ^{v_{b}}
$$

If the integration limits are substituted into (16), we get:

$$
\ln t_{b}-\ln t_{o}=\ln \vec{v}_{b}-\ln \vec{v}_{0}
$$

After the transformation, it is easy to write for this:

$$
\ln \frac{t_{b}}{t_{0}}=\ln \frac{\vec{v}_{b}}{\vec{v}_{0}}
$$

A simple and interesting relationship follows:

$$
\frac{t_{b}}{t_{0}}=\frac{\vec{v}_{b}}{\vec{v}_{0}}
$$

This is the change in velocity with age. At the time of the greater age $t_{b}$, the velocity $\vec{v}_{b}$ is also greater. At the beginning, the change in momentum was set to zero. The momentum must therefore be preserved. This is no longer evident from (19). Entropy constancy applies to mass decay. With $m_{0} t_{0}=m_{b} t_{b}$ can be written for equation (19):

$$
m_{0} \vec{v}_{0}=m_{b} \vec{v}_{b}
$$

This also means that the intermediate steps made in this derivation are plausible. One must note with (20) that we are dealing with one and the same object whose momentum remains constant with the change in mass. The object becomes faster with reduced mass and slower with increased mass. There are various causes for the occurrence of mass changes, e.g. mass acceleration due to gravitation.

Two masses are supposed to be in a system in which they are only exposed to their mutual gravitation.

Both impulses can be calculated according to equation (20) and are added together. The total momentum of the system is to become zero in the initial state via the velocities. This is the case at the reversal point after the two masses have moved apart as a result of a completely elastic collision. Both objects have their rest mass there. The masses are at rest for a short time, then the velocities change direction. The masses fall towards each other.

$$
m_{10} \vec{v}_{10}+m_{20} \vec{v}_{20}=0=m_{1 b} \vec{v}_{1 b}+m_{2 b} \vec{v}_{2 b}
$$

From this follows directly the relativistic conservation of momentum in one spatial direction:

$$
m_{1 b} \vec{v}_{1 b}=-m_{2 b} \vec{v}_{2 b}=\frac{m_{1 a}}{\sqrt{1-\frac{v_{1 b}{ }^{2}}{c^{2}}}} \vec{v}_{1 b}=-\frac{m_{2 a}}{\sqrt{1-\frac{v_{2 b} c^{2}}{c^{2}}}} \vec{v}_{2 b}
$$

The general validity of the conservation of momentum of objects could thus be traced back to universal mass decay.

\section{The Principle of the constant Number of Effects and Gravitation}


It is to be investigated for a two-mass system whether the principle of constant number of effects $m t=$ const. is also valid for gravitation. For this purpose, the gravitational energy in the free fall of two masses from rest and a certain distance until the collision of their surfaces is used.

The gravitational energy released until the two masses come into contact can be calculated as follows (see Figure 2).

$$
E_{\text {grav }}=\int_{r_{1}+r_{2}}^{r} F_{g r a v} d s=-\int_{r_{1}+r_{2}}^{r} \gamma \frac{m_{1} m_{2}}{s^{2}} d s=\left.\gamma \frac{m_{1} m_{2}}{s}\right|_{r_{1}+r_{2}} ^{r}
$$

The integration limits are defined here from the centre distance of both masses before free fall to the contact of their surfaces.

As an attractive force, the gravitational force has a negative sign. With the definition of the value $r_{b}=$ $r(r-h) / h$, where $\mathrm{r}$ is the centre distance and $h$ the surface distance, the gravitational work follows:

$$
E_{\text {grav }}=\gamma m_{1} m_{2}\left(\frac{1}{r}-\frac{1}{r_{1}+r_{2}}\right)=-\gamma m_{1} m_{2} \frac{h}{r(r-h)}=-\gamma \frac{m_{1} m_{2}}{r_{b}}
$$

As released energy, gravitational energy is also negative, because one has to expend positive energy to separate two masses from each other.

For each of the two masses, the principle of the constant number of effects should also apply (see equation (08)):

$$
m_{0} t_{0}=m_{b} t_{b}
$$

From both sides of this equation the term $m_{0} t_{b}$ can now be subtracted:

$$
m_{0} t_{0}-m_{0} t_{b}=m_{b} t_{b}-m_{0} t_{b} \quad \text { or } \quad-\Delta t m_{0}=\Delta m t_{b}
$$

Consequently, for both masses it can be written:

$$
\frac{\Delta m_{1}}{m_{10}}=-\frac{\Delta t_{1}}{t_{1 b}} \quad \frac{\Delta m_{2}}{m_{20}}=-\frac{\Delta t_{2}}{t_{2 b}}
$$

The gravitational energy (potential energy) from the state of rest of both masses until the collision of the surfaces must correspond to the negative sum of the kinetic energies of both masses:

$$
\begin{aligned}
& E_{\text {grav }}=-\left(E_{\text {kin } 1}+E_{\text {kin } 2}\right)=-\left(\frac{m_{10} c^{2}}{\sqrt{1-\frac{v_{1}^{2}}{c^{2}}}}-m_{10} c^{2}+\frac{m_{20} c^{2}}{\sqrt{1-\frac{v_{2}^{2}}{c^{2}}}}-m_{20} c^{2}\right) \\
& E_{\text {grav }}=-\left[\left(m_{1 b}-m_{10}\right) c^{2}+\left(m_{2 b}-m_{20}\right) c^{2}\right]
\end{aligned}
$$

For equation (29) can be written simply with (27):

$$
E_{\text {grav }}=-\Delta m_{1} c^{2}-\Delta m_{2} c^{2}=\frac{\Delta t_{1}}{t_{1 b}} m_{10} c^{2}+\frac{\Delta t_{2}}{t_{2 b}} m_{20} c^{2}
$$

It could be proved that the derived time dilation according to equation (31) for all relevant important experiments give better results on average than the time dilation of the theories of relativity [8].

$$
\frac{\Delta t_{1}}{t_{1 b}}=-\frac{\gamma m_{2}{ }^{2}}{c^{2} r_{b}\left(m_{1}+m_{2}\right)} \quad \frac{\Delta t_{2}}{t_{2 b}}=-\frac{\gamma m_{1}{ }^{2}}{c^{2} r_{b}\left(m_{1}+m_{2}\right)} \quad\left(t_{b}=t_{0}+\Delta t\right)
$$

If these time dilations are substituted into (30), one arrives again at (24).

This proves the principle of the constant effect according to (25) for the gravitation of a two-mass system. For the gravitational energy (24) of the two-mass system only comes out if one uses the equations (27) 
derived from this principle. The principle of constant action thus becomes an integral part of the quantum physical description of gravitation and allows it to exist in the first place.

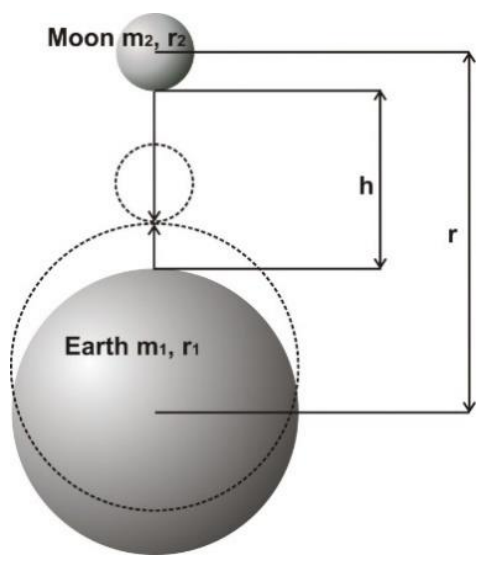

$\begin{array}{ll}\text { Legend } & \\ m_{1} & \\ m_{2} & 7.97710^{24} \mathrm{~kg} \\ r_{1} & 6.35210^{22} \mathrm{~kg} \\ r_{2} & 1.73810^{6} \mathrm{~m} \\ r & 3.84410^{6} \mathrm{~m} \\ h=r-r_{1}-r_{2} & 3.76310^{8} \mathrm{~m} \\ \gamma & 6.67310^{-11} \mathrm{~m}^{3} /\left(\mathrm{kg} \mathrm{s}^{2}\right) \\ v_{1} & 1.19910^{2} \mathrm{~m} / \mathrm{s} \\ v_{2} & 9.75010^{3} \mathrm{~m} / \mathrm{s}\end{array}$

Figure 2: Gravitational effect between two masses using the example of Moon-Earth falling on each other from their present distance and assumed rest position.

\section{The Explanation of astonishing Formulae}

Do the following formulae (32) and (33), which I found, cause us problems in explaining them within the framework of known physics [10.]? Yes, and huge ones at that, because they are correct and reveal mass decay. Apart from the age of space, only constants appear in the formulae according to today's understanding of the world. And what is even worse: every physicist, engineer, student and high school graduate can insert numbers and find that the formulas are correct.

Can this be a coincidence? Only if you assume that there is also a certain probability of your grandmother tunneling through the wall of your house alive. With both formulas the space age $t_{\text {uni }}$ comes out and of course that has to grow. So either we ignore the matter and accept that we can no longer sleep peacefully, or we take it seriously. Then we have no choice but to acknowledge that masses get smaller with space age and the gravitational constant grows quadratically with space age. All other possibilities of changing natural constants have been meticulously investigated and do not give a conclusive overall picture: the speed of light and Planck's constant must remain constant.

$$
\begin{aligned}
& t_{\text {uni }}=\frac{2 \hbar^{2}}{\gamma c m_{e} m_{p}^{2}} \\
& t_{u n i}=\sqrt{\frac{\hbar^{3} r_{p}}{\gamma^{2} c m_{e}^{2} m_{p}^{3}}} \\
& \hbar \quad \text { reduced quantum of action } \\
& \gamma \quad \text { gravitational value } \\
& c \quad \text { speed of light }
\end{aligned}
$$

\section{Summary}

Dark energy does not exist because space is not accelerating, but only expanding at a constant speed. Universal mass decay brings about a completely new world view that includes time as a variable physical quantity in all considerations. It could be shown that the principle of the constant number of effects, which can also be described as entropy constancy, occurs in mass decay as well as in gravitation. Equations (32) and (33), which were first put up for discussion by me in Research Gate on 2 December 2020, can be explained perfectly with mass decay. 


\section{References}

[1.] Gimsa, A., The Metric of Space-time, English-German, 2nd edition, p.29-43, published by the author, Potsdam, Germany, 2020, ISBN 978-3-00-064784-0

[2.] Gimsa, A., Development of the Distance Earth-Moon, International Journal of Scientific Research and Management, Volume 08, Issue 03 March 2020, DOI: 10.18535/ijsrm/ v8i03.aa01

[3.] Gimsa, A., The Expansion of the Universe, International Journal of Scientific Research and Management, Volume 08, Issue 07 Juli 2020, DOI: 10.18535/ijsrm/v8i07.aa01

[4.] Hubble, E., A relation between distance and radial velocity among extra-galactic nebulae, Proceedings of the National Academy of Sciences, Vol. 15, No. 3, March 15, 1929, S. 168-173

[5.] See Source [1.], p.26

[6.] See Source [1.], p.73

[7.] Gimsa, A., The beauty of nature, 2nd edition, p.5, Gieselmann Druck- und Medienhaus, Potsdam, Germany, 2014, ISBN 978-3-923830-94-7

[8.] See Source [7.], p.31

[9.] Gimsa, A., Dual mass systems under the infuence of gravitation, published by the author, Potsdam, Germany, 2017, ISBN 978-3-00-057218-0

[10.] See Source [1.], p.84 